\title{
Un homme à distance: essai sur le roman épistolaire contemporain
}

Fathéya Al-Fararguy

Faculté de Pédagogie-Université de Tanta, Égypte.

DOI:

رجل من بعيد: دراسة عن رواية المراسلة المعاصرة

بعد دراسة رواية ألباما سونج التى تعد رواية معقدة، ولكنها تبرز أهمية الكتابة تأتى رواية تقوم بنيتها على الرسائل. و لاستخلاص الجانب البلاغى وخصائص هذا النوع القديم ندرس رواية رجل ولئ من بعيد التى لاقت نجاحًا مدويَّا للكاتبة

.Katherine PANCOL

و أتناول فى بداية البحث نبذة تاريخية سريعة عن هذا النوع فى القرن السابع عشر والثامن عشر. ففى القرن التاسع

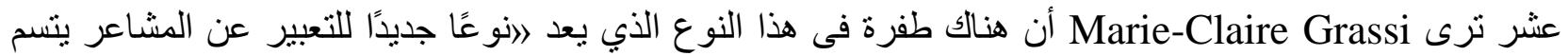

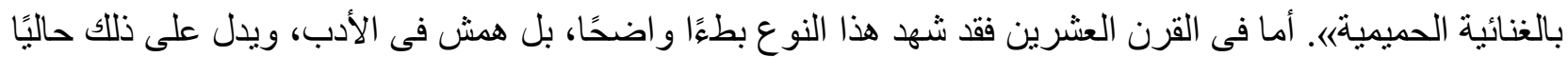

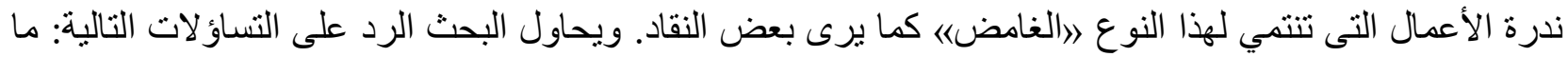

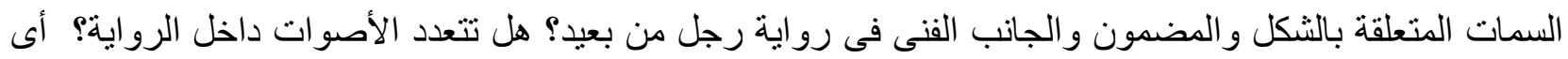
نوع من القراء تخاطب الرواية؟ هل ستعرف الرواية بالمراسلة ازدهارها أم اختفاءها بعد تطور وسئه وسائل الاتصال الحديثة: نوع

وللإجابة على الأسئلة السابقة يرتكز تحليل البحث على النقاد التالى اسماؤهم: F. Calas, S. Gruffat, G Genette, V. Jouve

وتركز الرواية على الكتابة بين متر اسلين: فكل واحد منهما مرسل ومستقبل. وتقوم فكرة البحث على خطة مكونة من

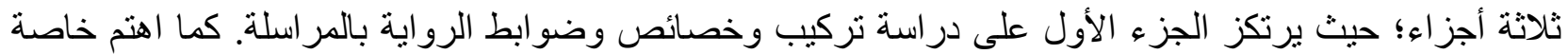

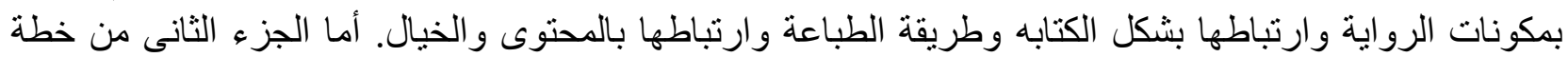

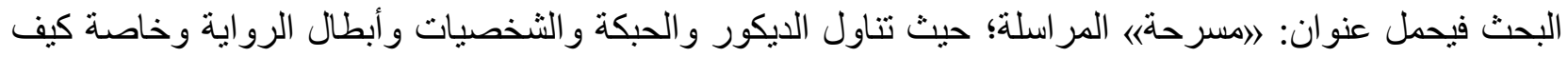

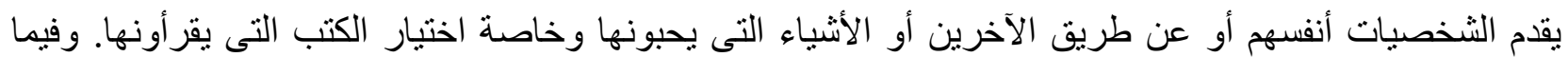

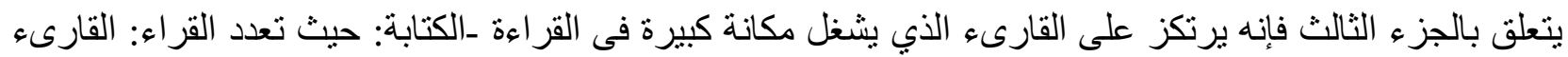

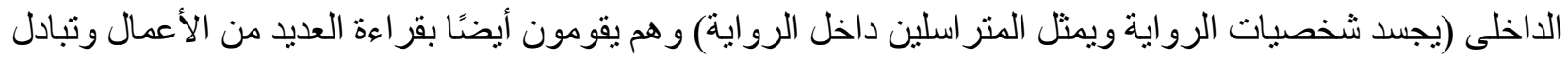
الآر اء حولها. و القارى الخد الخارجى (الذي يقوم بقر اءة الرواية).

و هكذا يبرز البحث تفرد وأصالة الكاتبة K. PANCOL التى تتضح عن طريق الاستمرارية والترابط الناتج عن

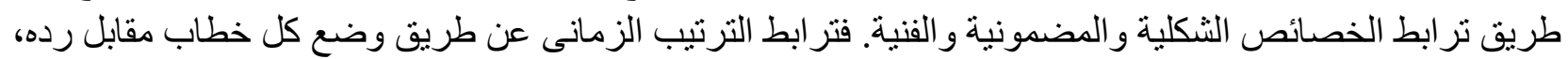

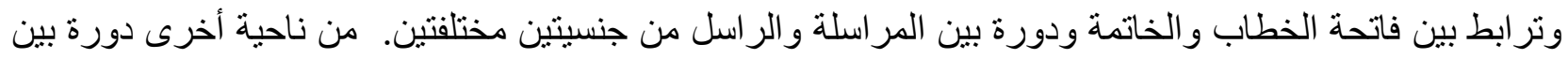

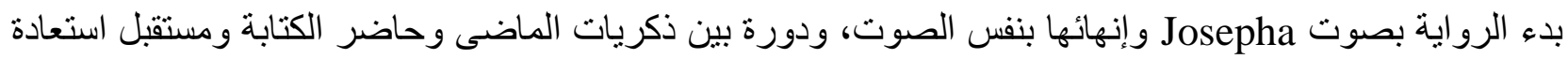
العلاقات ورد فعل قر اء المستقبل.

إن رحيل Jonathan غير المنوقع، وتنكره واستخدامه الخطاب كقناع، واستغلال المكان لاستعادة ذكريات الماضى وختاما إعلان الاعتر اف بواسطة افلام إنجليزية وفرنسية جعلت النسيج الروائى متر ابطًا.

Mots clés: Roman épistolaire, le péritexte, l'exorde, le décalage temporal. 
Roman par lettres ou en lettres ou en forme de lettre ${ }^{\mathrm{i}}$, roman épistolaire ${ }^{\mathrm{ii}}$, ou courrier du cœur ${ }^{\mathrm{iii}}$ est une des formes de la littérature intime ${ }^{\mathrm{iv}}$ ou bien la littérature des profondeurs ${ }^{\mathrm{v}}$. Vue comme forme de "discours", ce roman se fonde sur un schéma de communication entre un destinateur et un destinataire. Cette structure communicationnelle rend la lettre une action comme la parole qui vise à influencer le destinataire afin de créer un état nouveau et ouvre la voie de l'introspection et de la vraisemblance selon les termes de F. Calas (pp.9, 13, 16). Cet échange apparaît alors comme le substitut des paroles $^{\mathrm{vi}}$. Par ailleurs, la lettre forme un fait social et littéraire par le va et le vient entre deux pôles: le public et le privé (F. Calas, p.11).

Les particularités du roman épistolaire attirent notre attention pour l'analyse de son statut à nos jours. Au delà de l'identité formelle extérieure, ce genre envisage l'existence d'un noyau commun aux différentes formes de cette production ${ }^{\mathrm{vii}}$. C'est ce que Jean Montenot identifie par «protéiforme», la lettre étant à son avis «un objet difficilement identifiable du point de vue littéraire» viii . Aussi pouvons nous soutenir l'opinion qui estime que le roman en lettres se révèle capable de notations plus fines, plus vraies, plus logiques, plus synthétiques que le roman traditionnel, et permet de surmonter l'artifice des paroles: «écrire n'est pas seulement traduire une expérience, c'est aussi exister en inaugurant un nouveau rapport au monde, dont l'écriture n'est pas la méditation, mais l'expression ${ }^{\mathrm{ix}}$.
A partir du XVII ${ }^{\mathrm{e}}$ siècle, la création romanesque épistolaire voit le jour grâce au développement de la correspondance authentique privée. $\mathrm{M}^{\mathrm{me}}$ de Sévigné par sa condition de femme de pouvoir offre «la référence privilégiée» (S. Gruffat, pp. 21-22), le meilleur exemple de ce genre en tant qu'exercice littéraire et artistique. Le XVIII ${ }^{\mathrm{e}}$ siècle est une remarquable floraison illustrée par plusieurs chefs-d'oeuvre: Les Provinciales de Pascal, Les lettres philosophiques de Voltaire, Les Letttres persanes de Montesquieu, Lettre sur les aveugles ou Les Lettres à Spohie Volland de Diderot, La Nouvelle Héloïse de Rousseau ou Werther de Goethe. C'est à partir du $\mathrm{XIX}^{\mathrm{e}}$ siècle que naît, comme le constate Marie-Claire Grassi: «une nouvelle expression des sentiments caractérisée par le lyrisme confidentiel $\gg^{\mathrm{x}}$. Quant au $\mathrm{XX}^{\mathrm{e}}$ siècle L. Versini souligne que la vague de ces codes du bon usage épistolaire se ralentit (L. Versini p. 30). De même, F. Calas estime que ce genre épistolaire n'existe plus que dans les marges de la littérature. (F. Calas, p. 12).

En fait, nous avons trouvé une grande difficulté à trouver un roman épistolaire contemporain. Les romans en lettres sont très rares mais Un homme à distance $^{\mathrm{xi}}$ de Katherine Pancol a attiré notre attention. Ce roman préféré de la romancière serait «un signe, un signal, un appel...» ${ }^{\mathrm{xii}}$ Signe car ce roman est en premier destiné à communiquer avec quelqu'un, à faire savoir quelque chose (Petit Robert) et "signal" puisqu'il indique à chaque correspondant le moment d'agir et de répondre à l'autre. Ce jeu concentrique est bouclé par un appel et cet appel, des mots écrits, 
peut avoir la valeur d'une voix invitant à venir à soi donc à se connaître et à se raconter.

Le roman épistolaire impose certaines problématiques puisque l'intrigue est composée de lettres. C'est pourquoi S. Gruffat estime que le roman épistolaire s'incarne en une forme hybride et ambiguë. Ceci est dû à l'absence du narrateur extérieur «Le plaisir de la lecture tient dès lors à ce jeu de cache-cache qu'il s'agit de décrypter mais aussi à la découverte de psychologies diverses » ${ }^{\text {xiii }}$. Dans sa conclusion, S. Gruffat constate qu'il s'agit d'un «genre flou dont la définition et le statut dépendent d'un état de civilisation (S. Gruffat, p.115).

L. Versini estime que la problématique de ce genre réside dans l'esthétique du discontinu et l'intermittent. La discontinuité est dûe soit à un écart temporel d'une lettre à l'autre, soit au sujet traité qui impose un problème au roman épistolaire. Ainsi, parfois le destinateur traite d'un sujet, mais le destinataire, lui, répond à un autre sujet. A ce moment, vient le rôle du lecteur invité à une participation active en comblant ces lacunes.

Par ailleurs, Alain Viala indique que malgré l'abondance de l'héritage culturel, cette littérature épistolaire connaît au $\mathrm{XX}^{\mathrm{e}}$ siècle, une double restriction qui entretient mutuellement: le déni de "la qualité de l'art" et la réduction au roman par lettres. D'autres comme G. Lanson rejette le genre épistolaire hors du domaine littéraire. Une autre restriction réside dans l'usage périmé de cette forme d'écriture au temps de l'expansion des médias audiovisuels ${ }^{\mathrm{xiv}}$.
L'analyse d'Un homme à distance de K. Pancol soulève ainsi plusieurs questions auxquelles nous essayerons de répondre au cours de notre étude: Quelles sont les caractéristiques formelles, thématiques et esthétiques d'Un homme à distance? Ce roman est-il monophonique ou polyphonique? A quel type de lecteur s'adresse-til? Ce genre épistolaire connaît-il actuellement son épanouissement ou son déclin en particulier après le développement des moyens de télécommunication moderne (S. M. S, courrier électronique)?

Pour répondre à ces questions il serait peut être intéressant d'examiner ce que pensent les critiques contemporains du genre épistoliare. Nous nous référons souvent aux ouvrages de F. Calas, S. Gruffat, G. Haroche-Bouzinac, L. Versini et J. Montenot.

\section{Un homme à distance: structure et caractéristiques}

Le roman, se compose de 52 lettres, rédigées entre le 22 octobre 1997 et le 7 novembre 1998. Cette correspondance est précédée de deux pages d'une voix narratrice, celle de Josepha l'amie et la voisine de Kay, qui présente le roman et le clôture par trois pages qui fournissent au lecteur des informations sur le sort des deux épistoliers: Kay et David alias Jonathan.

L'analyse de la trame épistolaire et des parties constitutives de la lettre permet de savoir si la forme épistolaire est adéquate au message transmis par le roman. Selon S.Gruffat, l'écriture de la lettre, basée essentiellement sur l'exorde, la 
narration et la conculsion, quelle que soit lidentité des correspondants, obéit à des " normes formelles » et à « des règles de la rhétorique » (p.15).

\section{A. Le péritexte, la dimension matérielle de la lettre et sa typographie}

Dans Poétique, G. Genette définit le péritexte comme première catégorie spatiale qui comprend le nom de l'auteur, le titre, la dédicace, les titres de certains chapitres ou certaines notes, et l'épigraphe $\mathrm{x}^{\mathrm{xv}}$.

Le titre du roman - Un homme à distance invite à la découverte de la correspondance de cet homme qui vit loin. Le mot distance avec toutes ses connotations de l'écart, de l'éloignement contribue à l'émergence de l'intrigue. Le titre justifie donc la forme épistolaire $\mathrm{du}$ roman où la lettre est «le palliatif de l'absence » (F. Calas, p.74). Pour S. Gruffat cette distance facilite l'épanchement de l'être ${ }^{\mathrm{xvi}}$. K. Pancol attire notre attention par ce titre qui s'annonce par l'indéfini et incite le lecteur à se poser certaines questions: Qui est cet homme? A qui s'adresse-t-il? Que raconte le roman? Ce titre "littéral" xvii renvoit alors directement au sujet central.

En outre, le péritexte indique la modalité de la découverte de l'œuvre où réside l'essence de la fiction romanesque. Nous remarquons que dès le péritexte, apparaît l'effacement de l'auteur. Un homme à distance s'ouvre avec le dépôt d'une grosse liasse de lettres sur la table du restaurant de Josepha qui attire l'attention du lecteur au «pouvoir des mots» de son amie Kay et éveille sa curiosité pour découvrir le secret des lettres. Josepha a une fonction narrative explicite $^{\mathrm{xviii}}$ par son annonce liminaire «Ceci est l'histoire de Kay ». Selon F. Calas, cette "voix de la fiction" est un "code de lecture". C'est elle qui se charge de nous livrer "le secret d'origine" produit un « discours sur un autre discours, une fiction sur une fiction » (p. 111). C'est elle donc qui noue le pacte de lecture, précise la nature et l'objet du récit (histoire relatée en lettres) et informe le lecteur sur le personnage principal « Kay est ma voisine, mon amie, Kay est plus que ça encore...» ${ }^{\mathrm{xix}} \mathrm{C}^{\prime} \mathrm{est}$ la voix de la sagesse et de la philosophie, à l'incipite du roman, elle voit la souffrance magnifque en disant: «Bad things, sad things have to happen...sometimes » (U.H.D, p.8). A la fin du roman, cette même voix, chargée de l'ouverture, explique implicitement l'intention de la romancière: « La souffrance, c'est magnifique, quand on en guérit, quand on la transforme en compassion» (U. H. D., p. 152). Mais cette narratrice extradiégétique-hétérodiégétique (V. Jouve, p.26.) n'apparaît pas sur la scène de l'échange épistolaire, Kay se contente de rappeler ses efforts inlassables pour la sauver de son mal.

Dans Un homme à distance, nous ne trouvons pas d'épigraphe ou de dédicace. Par contre K. Pancol avant de terminer son livre par une page de remerciements adressés à plusieurs personnes, elle suggère à son lecteur, comme 
l'a déjà fait son personnage Kay la libraire, une longue liste de vingt-un livres -dont quatre ont la forme épistoliare-, intitulée: "Les livres de Kay et de Jonathan qui seront peut-être les vôtres". Cette invitation à la lecture est annoncée également par l'illustration de la couverture représentant un entassement figurant des livres volumuneux aux couvertures de couleur rouge, jaune, violet, bleu ciel avec leurs papiers blancs et jaunes, avec une marque page vers la moitié du livre rouge.

La typographie attire notre attention par l'emploi de l'italique pour les citations et les mots étrangers. Parfois nous rencontrons certains mots anglais qui nous rappelent qu'il s'agit d'un américain et l'origine anglaiseitalienne de Kay. Signalons également que la typographie de deux épistoliers a une forme différente. «Les personnages utilisent l'écriture comme substitut. Les mots prennent la place des corps absents, ils viennent combler le vide que l'absence a créé ${ }^{\mathrm{xx}}$. Kay intéressée par ce client "connaisseur" et cultivé, lui écrit à la main. Jonathan, lui, écrit sur ordinateur en commentant: "J'espère que vous ne vous formaliserez pas (...) Je suis si habitué au clavier que mes doigts sont gourds quand ils tiennent un stylo» (U. H. D., p. 13). Jonathan représente la modernité, mais l'absence de la mise en scène de l'arrivée et du départ du courrier nous approche un peu de l'ère du règne de l'internet.
La dimension matérielle de la lettre reflète la personnalité de l'épistolier. La lettre est un corps présent habillé par une enveloppe, ellemême décorée de timbres, de tampons, des adresses du destinateur et du destinataire et des dates marquantes de leurs vies. Mais ce qui nous intéresse le plus c'est l'arrière fond culturel assez riche qui double les lettres de Kay et Jonathan.

\section{B. L'exorde, la conclusion et la signature}

Les formules introductives et finales traduisent le degré d'intimité entre les épistoliers. En haut à gauche de la lettre, le prénom, le nom et l'adresse du destinateur sont marqués. S. Gruffat considère que «L'exorde a en effet pour fonction d'opérer une captio benevolontia, c'est-à-dire de susciter la bienveillance et l'intérêt de l'interlocuteur» (p.15) Les épistoliers d'Un homme à distance usent parfois des formules d'appel: "Monsieur", "Mademoiselle", ou de leurs prénoms ou commencent leurs lettres directement $^{\mathrm{xxi}}$. Le ton devient plus amical presque vers la moitié du roman. Jonathan prend l'initiative en appellant son épistolière par son prénom à l'occasion de la nouvelle année. Dans sa vingt-et-unième lettre adressée de l'Hôtel "Le Pigeonnier", il écrit «Chère Kay» (p. 87) dans sa vingt-sizième lettre «Très chère Kay» (p. 112) puis dans la vingthuitième lettre «Ma chère Kay» (p. 116) et elle, à son tour, répond par «Cher Jonathan» (p. 88). 
Quant à la fin de la lettre, elle constitue selon S. Gruffat, qui met l'accent sur la préoccupation fondamentale de l'émetteur, un témoignage: «d'affection», «de remerciements », «d'une marque de référence», ou un «congé»(p.17). Elle prend plusieurs formes entre le destinateur et le destinataire en lui rappelant la nature du lien qui les unit et donne, par là, une sorte de «circularité» ou d'un «mouvement en boucle» (G. HarocheBouzinac, p.21) entre l'émetteur et le récepteur. Dans la première lettre adressée par Jonathan à Kay, la formule de politesse marque une grande distance entre les deux correspondants. Mais Kay initie sa deuxième par une formule amicale. D'autres exemples révèlent une ceraine familiarité qui s'établit graduellemet entre les deux épistoliers. Parfois, les deux épistoliers se répondent de la même manière: «Comme un mois de décembre $»(U . H . D$, p.34) écrit Kay, «Comme un jour de Noël» (U. H. D, p. 35) répond Jonathan. L'épistolière a recours à une durée plus longue "mois", alors que l'épistolier utilise une durée plus courte "un jour" (notions temporelles). L'emploi fréquent d'adverbes de manière"en ment" comme amicalement, boudeusement ... etc vont dans le même sens.

La familiarité se traduit également par les formes des signatures: certaines missives signées de Kay Bartholdi, de Jonathan Shields, ou de leurs prénoms seulement et d'autres sont sans signatures.
Par ailleurs, nous remarquons que dans certaines lettres, les épistoliers ont souvent recours au post-scriptum. Ces courts messages annexés rajoutés après les signatures peuvent marquer soit un oubli ou l'ajout d'une information non liée directement au message soit mettre un point particulier en avant, ou souligner un point ou plutôt un moment d'hésitation pour avouer une chose. Le postscriptum (PS) est utilisé dix fois: six fois dans les lettres de Jonathan (U.H.D, pp.13, 21, 28, $52,60,84,98)$ dont le plus long PS est celui de la page 98 dans lequel il décrit le propriétaire de l'hôtel et quatre fois chez Kay (U.H.D, pp.63, 66, 83, 111). Jonathan utilise les PS plus que Kay parce qu'il essaie de dévoiler graduellement sa vraie identité.

Les voix des épistoliers traduites par l'exorde, la conclusion et la signature produisent un effet de retenissement plus profond par le choix des pronoms.

\section{Le style et les pronoms de la communication épistolaire}

A. Viala compare la conversation et la lettre et note leurs similitudes fondamentales ainsi que leurs différences. Dans les deux cas, la communication s'effectue par un message verbal. Cet acte de communication met en œuvre une pragmatique «pour agir sur (le recepteur), pour le toucher ou le convaincre, pour estomper les effets de son absence» (S. Gruffat, p.4). Le locuteur exerce ainsi une action sur son auditeur /lecteur pour l'informer, 
l'émouvoir ou le convaincre. En compensation des gestes, des attitudes, des intonations et de tous les signes physiques, la lettre affiche plus de contraintes conventionnelles ${ }^{\mathrm{xxii}}$.

A la lecture d'Un homme à distance, nous ne trouvons pas d'épistoliers épisodiques. Le choix de la forme épistolaire repose sur une observation intérieure entre deux destinataires. Le choix de duo épistolaire donne naissance à un discours "personnel", "intime" et crée un espace d'une auto-analyse, d'une volonté de comprendre les reflexions de cet emetteur épistolier et d'établir une relation logique entre ses paroles et ce que le moi recepteur éprouve $\mathrm{x}^{\mathrm{xxiii}}$. Ceci nous amène à constater qu'il démontre la construction psychologique par « l'examen de conscience, ou le discours frotté à la parole de l'autre» (F. Calas, p.91). Ces voix intérieures, attribuent à chaque épistolier son propre ton «qui permet de le singulariser » (S. Gruffat, p.3) ou comme le décrit L. Versini « une voix qui vibre dans le silence» (p. 44) qui a tout le temps de sonder son moi profond. F. Calas voit que «Le discours de soi à l'autre devient discours de soi à soi »(p.75). Le moi se dévoile devant l'autre et incite à réfléchir sur certains sujets. De cette manière, le lecteur extérieur du roman semble pénéter dans le huis clos de chacun de deux correspondants.

L'écriture épistolaire s'aiguise sur les pronoms: je, vous, tu. L'emploi de la première personne donne une vivacité au roman et nous mène vers l'écoute attentive de la voix de l'épistolier qui par ce choix cherche à s'imposer. Les événements sont teintés ainsi par une instance individualisée, par conséquent l'œuvre paraît comme une non-fiction (Cf. F. Calas, p.111). Ecoutons Kay qui expose ses sentiments et sa détresse à la première personne: «Je rêvais que tu entres, un jour, dans ma librairie, que tu me prennes par la main et que tu m'emmènes n'importe où» (U.H.D., p.145).

Le choix des pronoms vous ou tu, montre le degré de rapprochement entre les épistoliers. Au début, ils se vouvoient puis ils se tutoient « Kay je t'en supplie. Dis-moi de venir » (U.H.D, p.133). Le recours au tutoiement donne ainsi aux lettres un ton plus familier. Ce ton rend l'écriture plus libre. Cette familiarité augmente le rapprochement entre les épistoliers, à tel point que les caractéristiques marquantes d'une lettre amoureuse s'accentue par le vocabulaire affectif, les hyperboles et la métaphore. Kay parle de son métier en disant: «Et je me suis construit ma bulle » (U.H.D, p.145). Le métier assimilé à une bulle forme une métaphore qui connote sa fragilité. Ce globule repli d'air ou de gaz peut facilement éclater et disparaître. Mais quand Kay et Jonatahn sont sur le point de se quitter et de rompre cette relation, par suite cette correpondance, ils reutilisent le pronom "vous" signe de l'éloignement prochain dans les cinq dernières lettres.

Signalons par ailleurs que K. Pancol exlpoite d'autres procédés qui peuvent impliquer 
fortement le destinataire comme: l'apostrophe, l'exclamation, la répétition et l'interrogation « Oh Kay! Pardon, mille fois, dix mille fois pardon, cent mille fois, un million de fois pardon! »(U.H.D, p.67). Dans sa vingt-etunième lettre, l'épistolière répète "J'ai envie de vous écrire" quatre fois pour insister sur le sens: «J'ai envie de vous écrire parce qu'il fait beau. J'ai envie de vous écrire pour l'amour des mots. J'ai envie de vous écrire parce que vous êtes loin et muet! » (U.H.D., p. 99)

Il est à remarquer qu'au début de leur communication, Jonathan lui pose des questions sur la signification de certains mots en français comme: «insolent» et «impertinent» (U.H.D., p.21). Mais après quelques mois, il a changé de ton. En homme expert de la vie, il prend un ton de conseiller devant l'inexpérience d'une française imprégnée de sa profession. Il choisit ce trait de caractère pour se présenter: «Ce n'est pas pour autant que je suis un vrai "sage"» (U.H.D., p.11). Nous sentons que les lettres de Jonathan prennent une tournure didactique: il donne des conseils, des informations sur le monde: « Dites? Vous les lisez vraiment attentivement, mes missives! Chaque mot est compté, pesé, passé à la loupe! Je n'ai pas droit à la faute, au moindre écart d'humeur!» (U.H.D., p.58)

L'échange épistolaire se traduit également à travers les liens tissés entre les mots et la syntaxe. Comme l'émotion détermine le choix du temps, elle est également le pivot de la syntaxe: «Si l'épistolier est sous l'emprise de l'émotion, la syntaxe est, soit amplifiée par des énumérations, des répétitions, soit brisée par des anacoluthes ou des ellipses» (S. Gruffat, p.67).

L'écriture épistolaire familière peut contenir une syntaxe «incomplète », «elliptique » (S. Gruffat, p. 67). Fathi Sayed ajoute que par l'émotion la phrase est désorganisée, disloquée, rapide, hâchée et abonde en tours impératifs et en formes d'insistance ${ }^{\text {xxiv }}$. La forme épistolaire, de par sa nature émouvante, convient plus à l'écriture romaneque sentimentale: le personnage confiant ses pensées et sensations, en une lettre, entame un réel "portrait de l'âme"et établit avec son récepteur une communication transparente: « Je connais le huis clos. "Il n'y a qu'une atmosphère où l'amour n'étouffe pas, c'est la solitude"» (U.H.D., p.103)

La variation de la longueur de certaines lettres joue un rôle sur le rythme du roman. Quelques lettres se caractérisent de par leur style télégraphique: «Je lisais. Je lisais. (...) Peu à peu, le monde, autour de moi, s'effaçait, escamoté par les mots du livre. Je répétais des phrases, relisais des paragraphes, reposais le livre quand les mots étaient trop forts» (U.H.D., p.102). D'autres lettres ressemeblent à des SMS: (U.H.D., p.110, 111, 112, 113, 114, 115, 129, 130, 131). Ces formes brèves ou bien ce "jeu de cache-cache" selon le terme de S. Gruffat, peut interpèter une période de tension entre les deux épistoliers. Cette 
écriture semble beaucoup plus proche du courriel rapide chez les internautes. Kay est en position de destinateur parce qu'elle est la première à écrire. La longueur de certaines réponses, fait d'elle la protagoniste du roman. Mais personne ne prends en main le déroulement épistolaire. Elle a rédigé vingtsept lettres alors que Jonathan en a écrites vingt-cinq d'où l'équivalence dans la communication épistolaire.

\section{La théâtralisation épistolaire}

\section{A. Le décor de l'écriture épistolaire et le décalage temporel}

Le lieu de la narration est un élément de l'action (F. Calas, p.11). Les deux correspondants s'écrivent de lieux différents. Kay écrit toujours d'un seul lieu, mais Jonathan se déplace beaucoup. Elle expédie toutes ses lettres d'une même adresse, celle de sa librairie de Fécamp, Les Palmiers sauvages, qui se trouve 14, quai Maupassant. Le nom de la librairie est aussi le titre d'une œuvre de William Faulkner, livre préféré de Jonathan. La description de l'espace est désignée par ancrage (V. Jouve, p.40) puisque le sujet de la description est indiqué en tête de la lettre et dans le contenu de la première lettre de Jonathan. Cette description est introduite par un "personnage qualifié" et par "un verbe de perception" (V. Jouve, p.41) «(mon oil) a été attiré »(U.H.D., p.11). Cet espace structuré géographiquement par des indications situant la réalité décrite (haut/bas) attire le regard de
Jonathan, scénariste et producteur des films et ensuite son admiration. Au milieu des murs hauts et blancs, cette librairie se dresse avec son palmier, «toute droite, toute haute, d'un vert amande assez surprenant. Toute pimpante avec ces stores jaune et blanc, (...) face à la mer (U.H.D., p.11). Il en tombe amoureux à tel point qu'il pense en faire son: «port d'attache littéraire» (U.H.D., p.12).

La librairie et le foyer de Kay sont les deux noyaux principaux de son existence à Fécamp ${ }^{\mathrm{xxv}}$. Elle aime vivre dans cette ville avec ses rues étroites, ses petites maisons modestes semblables de briques rouges et de silex noirs et emboîtées les unes contre les autres: "Pour moi, un jour, le monde s'est arrêté à Fécamp » (U.H.D., p.113). Kay décrit son foyer ou son lieu personnel comme enchanteur parce qu'il donne sur le port, la ville et la mer. Elle jouit des embruns salés de la mer, de l'odeur de port et de poisson, de l'atmosphère et de la lumière. La mer est le berceau des rêves de Kay. Elle se lève très tôt avec le premier rayon de soleil « qui caresse le plancher d'un jaune pâle, froid et timide en hiver, chaud, gaillard et doré en été » (U.H.D., p.15). Tout l'enchante.

A tout ce qui précède s'ajoute "le cadre social" (L. Versini, p. 127), un cadre de vie de province, "enchanteur" comme dans le roman de Flaubert. A l'occasion de mariage d'un de ses lecteurs, elle décrit les mariages à Fécamp. Par l'intermédiare des reminiscnces littéraies (et nous pensons à la noce paysanne des 
Bovary) elle écrit: «Les mariages, ici durent deux jours plus! Et devinez quoi? On n'arrête pas de manger. Poisson et viande à chaque repas, et un trou normand entre chaque mets! $»($ U.H.D., p.95)

Quant à Jonathan, venu en France à la recherche des sites touristiques, il écrit de différents lieux comme Barfleur, Sète, Arles, et Aix-en-Provence. De ces lieux, le dernier forme un décor privilégié dont les deux épistoliers ont des souvenirs communs. Nous remarquons que sur le plan géographique, Jonathan parcourt la France du nord au sud et choisit des villes dont la pêche est l'activité économique principale. Ces différents déplacements accordent au roman un rythme rapide et bref qui convient au rythme du lecteur du XXI ${ }^{\mathrm{e}}$ siècle.

De tout ce qui précède, nous observons que la mer constitue un point d'attache pour les deux épistoliers: «Je m'arrête tout le temps... Pour regarder la mer qui râpe les rochers »(U.H.D., p.20) exprime Jonathan. Il décrit ses impressions sur certains lieux comme Barfleur: «l'endroit est si beau que je ne peux me résoudre à le quitter» (U.H.D., p.18). Cette ville riche d'un patrimoine de qualité est un des plus beaux villages de France. Il informe Kay sur les différences de goût entre les générations: $M^{\text {me }}$ Le Cozze, propriétaire de l'hôtel n'apprécie pas la qualité de la nourriture surgelée, alors que pour sa fille, cela facilite la vie.
D'après F. Calas: «Le motif du voyage joue donc un rôle double puisqu'il sert, d'une part, à justifier l'échange épistolaire en raison de l'éloignement qui se crée et d'autre part, à permettre la critique de la société européenne en introduisant le regard des explorateurs étrangers » (p.79). Attiré par la région d'Aixen-Provence, Jonathan invite Kay à le rejoindre. Il souhaite revivre le passé avec elle dont le point focal est l'Hôtel Le Pigeonnier d'où il envoie quinze lettres de la mi-juin au 7 novembre 1998: «La Provence et son arrièrepays sont des lieux de villégiature privilégiés par les Américains et je dois fouiller le pays à la recherche de villages et d'endroits "pittoresques"» (U.H.D., p.87). Pourtant l'endroit, en soi, est loin d'êre désagréable. L'hôtel est présenté par ses propriétés et il est mis en relation à la mer, au chant des cigales et aux glycines avec ses fleurs odorantes. Mais Kay s'interroge sur le choix spacial de ce lieu parce qu'elle sait qu'Aix-en-Provence contient de nombreux hôtels pour touristes américains. Ce choix la laisse «songeuse » (p.91), « nostalgique et faible! »(U.H.D., p.96) Elle réagit spontanément et nous invite à un retour en arrière. Elle ne peut rester au mutisme: « Alors là, vous le faites exprès! Je connais cet hôtel dont vous me parlez! (..) Je devais avoir vingt ans.» (U.H.D., p.88) Elle se souvient de ce moment après douze ans en écrivant: «Le temps s'évapore si vite, mais les souvenirs persistent» (U.H.D., p.88). 
Ce qui nous mène à nous poser cette question: comment se traduisent ces déplacements sur l'axe du temps?

Nous remarquons que la notion de l'espace dans ce roman est intimement liée à la problématique du temps. En tête des lettres, les épistoliers précisent les lieux à gauche et les dates à droite. Dans La poétique du roman, V. Jouve indique que l'aspctualisation de l'espace permet le retour vers des moments du passé donc un retrour aux souvenirs. Elle permet donc d'actualiser la relation entre les deux amoureux (Cf. V. Jouve, pp.42-44.). L'unité du temps est définie par la circularité des lettres soigneusement datées et par leur ordre chronologique. De plus, la cinquième lettre est la seule où on peut constater une indication horaire à côté de la date «Le 25 décembre 1997, dix-neuf heures» (p.36).

Dans Un homme à distance, les périodes de ruptures se produisent trois fois: la première séparation a lieu avec le départ de David, la deuxième dure un mois du 21 décembre 1997 au 20 janvier 1998 et la dernière survient après l'aveu de David. Durant la deuxième séparation, Jonathan ne répond pas aux lettres de Kay: «Je rageais, je bouillonnais, je dépérissais, je happais l'air par toutes mes branchies » (U.H.D., p.53) écrit-il. Cette lettre se construit sur l'isotopie du champ lexical de colère et de désepoir (grève, courir, furieux, rageais, bouillonnais, dépérissais). Le lecteur passe des confidences passionnées à des protestations brûlantes: « quand je lis vos mots, je vous trouve arrogant, impudent, agressif »(U.H.D., p.57). Quand il tarde à répondre, elle se livre à une satire pénétrante. Le point culminant de l'action éclate avec le souvenir de la violente séparation des deux épsitoliers. Elle a lieu quand Jonathan envoie six lettres, du 17 septembre au 26 octobre, sans recevoir de réponse de Kay. Elle ne lui répond que le premier novembre.

Entre la date d'envoi et la date de réception, l'émetteur, imagine la réponse et la réaction du récepteur. Ce temps d'attente est désigné par G. Haroche-Bouzinac comme un "tempslongueur"(p.77). L'idée de décalage temporel représente une source de jeux sur le temps « tout auteur de lettre sait que le présent de l'écriture correspond au futur de la réception, tout récepteur sait également que le présent de la réception renvoie au passé de l'expédition» (G. Haroche-Bouzinac, p.77). Cette circularité entre le passé des souvenirs, le présent et le futur des retrouvailles donne une sorte de continuité à l'écriture épistolaire.

La première lettre s'ouvre sur des verbes au passé composé qui signalent un contexte passé: «J'ai pris connaissance », « j'ai bien compris ». Kay a un recours flagrant à l'imparfait «j'étais». L'emploi du futur met l'accent sur la continuité discursive «Je noterai» toujours officielle. Mais dans les lettres suivantes quand la relation devient plus amicale et qu'on s'échange des propos intimes, le présent prend sa place dans la lettre. Cette mise en place actualise le temps de l'écriture « 
si le présent est évoqué, il est alors associé à la douleur de la solitude ou recréé grâce aux pouvoirs de l'imagination et de l'écriture. » (S. Gruffat, p.68). Pour V. Jouve, l'emploi du présent rend la narration "simultanée" (p.36.) et donne l'illusion que le narrateur écrit au moment même de l'action.

\section{B. Le cadre sérial de la lettre et la narration}

Les lettres ne sont pas numérotées mais rangées selon l'ordre de leur envoi et de leur réponse. L'action cislée dans la trame romanesque par la lettre accorde au roman épistolaire sa particularité. D'où se pose alors la question de savoir quelles relations sont établies entre l'acte de narration et le fait narré. Les événements racontés se passent au moment où ils sont vécus ou donnent-ils lieu à un récit rétrospectif et distancié? (Cf. S. Gruffat, p.98)

Dans Un homme à distance, chaque lettre a sa propre voix, son propre style, et sa propre pensée. Mais à quoi sert l'étude du classement des lettres? A cette question nous répond $\mathrm{G}$. Haroche-Bouzinac. Il estime que l'harmonie épistolaire émerge du classement de chaque lettre en face de sa réponse. Cette harmonie crée un effet d'entente, de l'établissemnt d'un écho, et de la compréhension mutuelle (p.6). Pour F. Calas, cet ordre rend la narration directe puisqu'elle est distribuée à chaque personnage dès qu'il devient épistolier (p. 43).

Dans les lettres de ce roman, on parle de vies personnelles, professionnelles et sociales. Or le lecteur constate qu'il y a une sorte d'affrontement entre la vie professionnelle de Kay en tant que libraire et celle de Jonathan scénariste et producteur de films à travers ses voyages. La profession de Kay libraire représente pour Jonathan une occupation «si désuète...» (p.13) Il s'interroge: « comment peut-on être libraire au presque XXI siècle? » (U.H.D., p.13), le ton ironique se traduit par ces mots: «Vous voilà libraire! Vous ouvrez pour la société! Vous sauvez des âmes, pansez des plaies. Sainte Kay, priez pour moi, veillez sur moi! »(U.H.D., p.23) Mais Kay, fière et heureuse se rejouit d'être libraire; elle répond à Jonathan qu'elle trouve un grand plaisir à exercer sa profession. Elle écrit: «Et pour répondre à votre question, être libraire n'est pas une "occupation" mais une profession» (U.H.D., p.15). A Noël elle prépare elle même les bandeaux des nouveaux livres qu'elle collera sur les livres et sur lesquels elle a marqué cette expression accueillante à la lecture: «si ce livre pouvait nous rapprocher de vous...»(U.H.D., p.12). Kay énumère les détails de son travail quotidien: pour être au courant des titres de l'actualité, elle lit tous les journaux pendant le repas, avant le sommeil et à ses heures de loisirs. Il est à mentionner que Kay avant d'être libraire, pendant son séjour parisien, elle travaillait dans une boîte de publicité où elle gagnait beaucoup d'argent. Mais par l'héritage paternel elle ouvre une librairie et préfère se retirer dans un espace assez limité pour nourrir son imagination. 
Dans ce roman nous avons les deux faces d'une seule monaie lecture-écriture. Manquant d'expérience, Kay commence à se former par ses lectures et dans le même sens elle aide et oriente ses clients. Elle est le réservoir de livres (libraire) de lectures qui nourrissent sa vie et alimetent son écriture. Cette libraire a: « La tête dans les nuages et les livres, les pieds dans les comptes et la poussière » (U.H.D., p.34). Un autre type de focalisation s'impose sur sa profession de libraire grâce à cette relation avec les clients/lecteurs de la librairie de Kay; c'est chez eux qu'elle trouve estime et reconnaissance pour ses efforts, elle devient une de leurs chers amis. D'autre part, ces lectures exploitées, citées et commentées dans la correspondance des épistoliers enrichisseraient tout lecteur curieux.

Kay sent qu'elle a un message envers ses clients: «Je voudrais leur enseigner le respect, mais, n'en ai guère le temps ni les moyens!» (U.H.D., p.33) A Jennifer, fille de la coiffeuse, âgée de quatorze ans; Kay conseille: Le Grand Meaulnes d'Alain-Fournier, Les Hauts de Hurlevent, Lettres d'une inconnue de Zweig, Ce que savait Maisie de James. La jeune fille en apprécie la lecture, devient plus motivée et son niveau s'est amélioré à l'école. La mère de cette jeune fille sauvée par les livres est profondément reconnaissante à Kay. « C'est pour ces moments-là que je me félicite d'être devenue libraire » (U.H.D., p.24). L'affectivité traduite par cette phrase montre l'attachement de Kay à sa profession. On la remercie pour l'excellence de ses conseils. A un autre client, elle recommande un roman d'amour afin d'avouer son amour à son amie: Amour de perdition de Camilo Castelo Branco. Ils se marient et l'invitent à leurs noces. Ces exemples montrent que la fiction et l'écriture peuvent jouer un rôle dans le changement de la vie d'un être humain.

Ainsi, trouvons-nous un défi au plan temporel entre le passé et le présent et au plan spacial entre l'Europe incarné par Kay et l'Amérique incarné par Jonathan. Rappelons à cet égard que K. Pancol a vécu à New York où elle suit des cours d'écriture à l'université de Columbia. Grâce à ce séjour, la romancière réussit à bien imaginer son personnage motivé par des ambitions et avide de gloire. L'Amérique où on aime l'art et le cinéma, diffère de l'Europe où on préfère la lecture et les librairies. «J'ai oublié de vous dire que je n'ai ni télé, ni magnétoscope, ni ordinateur! Il va falloir que j'investisse d'ailleurs... mais je n'en suis pas si sûre! Je préfère lire » (U.H.D., p.44) explique Kay à Jonathan. Les deux épistoliers sont ainsi des distributeurs du savoir. Elle, de par sa profession et lui de par ses voyages et son travail au cinéma. Il a le regard d'un étranger.

Kay se réfère aux préjugés populaires et compare les Français aux Américains: «on dit que les Français sont des Latins, qu'ils ont le sang chaud et qu'ils parlent trop parfois » (U.H.D., p.16) écrit-elle. En tant qu'Américain, Jonathan présente sa vision 
culturelle: «Les Américains considèrent les Français comme des drôles de hères, incohérents, fiers, rebelles, au sang chaud comparés à nous, Anglo-Saxons, plus froids et réservés. (...) Et les Français croient tous que les Américains sont riches, obèses et totalement incultes » (U.H.D., p. 20). L'opposition entre la nature de deux peuples: "froid", "chaud", "culte", "inculte" met les deux épistoliers face à leur réalité anthropologique. A cet égard, rappelons ce que Lucia Omacini écrit: «La lettre, qu'elle soit vraie ou fictive ou même intégrée à un cadre romanesque, traduit, grâce à ses formes mobiles, une démarche progressive de l'esprit, ralliant la critique à la recherche de la vérité ${ }^{x x v i}$. La conception de chaque peuple incarne l'affrontement entre les deux cultures. Si Kay représente la dimension culturelle, Jonathan figure le côté matériel.

\section{Kay et Jonathan: comment se présentent-ils?}

Le maniement de la lettre d'après chaque épistolier révèle sa personnalité. La lettre donne l'effet du miroir à trois aspects: « elle est un modèle », « un portrait », et « un lieu de réflexion ou de méditation ». A ces trois aspects correspondent troisintentions: « la direction morale », «l'épanchement du moi » et «la naissance de réflexions» (G. HarocheBouzinac p.97).

Dans sa troisième lettre, Kay annonce: «On pourrait même me traiter de méticuleuse ou de vieille fille!», «on m'appelait "la princesse au petit pois"»(p.14). Elle se présente aussi en tant que femme/enfant; confidence qui connote sa sensibilité et sa naïveté. Fille d'une mère anglaise et d'un père italien, Kay a vécu une enfance douleureuse. La mère fort bavarde, le père Guiseppe Bartholdi, violent, grossier, très ambitieux: «Une seule chose l'intéressait: faire de l'argent. Et la fibre paternelle ne le démangeait pas » (U.H.D., p.119). Nous observons que Kay n'a jamais mentionné le prénom de sa mère, elle se souvient d'elle en disant maman ou ma mère. Implicitement nous reconnaissons qu'elle n'a jamias joué un rôle influent dans sa vie. Kay a vécu dans une « tourmente infinie » à cause de ses parents « dissemblables », « effarés », «abasourdis », elle a grandi «en entendant leurs cris, leurs plaintes, leur fureur, leur douleur, les mains plaquées sur les oreilles pour ne pas écouter », ce qui l'amène à « se raccrocher à un troisième larron pour aspirer un peu d'air frais, d'amour, d'émerveillement » (U.H.D., p.77). Cette relation détermine ses rapports avec les hommes tout au long de sa vie: « Il coule dans le sang de mes veines la violence de mon père, sa rudesse, sa volonté féroce, une certaine cruauté même dont je ne suis pas si fière...Mais je saurai m'en servir si vous me menacez »(U.H.D., p.83) avoue-t-elle. Après la mort de sa femme qu'il maltraitait, le père épouse une de ses serveuses, Maria une sicilienne docile et rude. Kay et son frère Marco «collés comme des berlingots» 
(U.H.D., p.119) quittent la maison paternelle pour vivre dans l'immense studio de David Royle. Il leur apporte des livres leur apprenant la beauté des mots, l'importance du détail et la structure d'une histoire. Il devient un père notamment pour Marco. Il lui dessine un avenir flamboyant en l'initiant à de nombreuses disciplines comme le tennis, l'anglais, la boxe française, la danse de combat, la conduite.

L'inexpérience de Kay et son enfance malheureuse l'ont mise facilement sous l'emprise de David. Avec le souvenir d'une mère torturée par le père et des enfants laissés à la belle-mère, Kay veut éprouver son existence féminine.

Elle se jette aux pieds de David succombant aveuglément à ses sentiments. Relevant le défi, contre le monde entier, de mener une vie heureuse sous les auspices d'un étranger égoïste, ébloui par la gloire, le portrait de David est brossé par Kay. C'est un homme «drôle, raffiné, cultivé, séduisant, rapide, puissant» (U.H.D., p.41). Il est dominé par son amour pour ses ambitions, pour ses projets et pour la vie. En un mot, David Leroy comme son nom indique ses manières hautaines: «voulait être le roi du monde et dicter sa loi» (U.H.D., p.41). A quinze ans, Kay ne résiste pas à sa séduction. Selon ses termes, elle épouse ses rêves et ses ambitions.

En refusant le sort qui lui était socialement réservé; David a quitté Kay et son frère. Il les quitte soudainement pour réaliser sa gloire dans le domaine du cinéma, ailleurs. Kay s'interroge « Cet homme qui nous abandonnait. Pour qui? Pour quoi? Pourquoi, Jonathan? Pourquoi est-il parti? » (U.H.D., p.74) Devant la jeune fille âgée de quinze ans, David était «si pétrifié, si bouleversé » (U.H.D., p.135). Kay ressent de la fierté à cause de son amour pour Jonathan, à tel point qu'elle n'a ni honte ni peur d'établir sa liaison aux yeux de tous. Elle appréhende sa conscience individuelle et le monde extérieur avec confiance, sérénité, zèle, force et chaleur. «J'affrontais les regards dans la rue. Je marchais droite comme une allumette avec des yeux incandescents qui brûlaient les mauvaises langues. Je fixais les commerçants en faisant les courses de la maison, de "notre" maison » (U.H.D., p.136). Toutefois Kay "allumette" s'est éteinte avec le départ de Jonathan. Marco et Kay étaient complétement perdus et bouleversés. Il travaille dans une maison de production de cinéma sous le nom de Mark Bartholdi. Marco devient producteur indépendant. La réussite de Marco, sa célébrité et son absence au niveau des relations familiales ressemble à un grand degré celle de Jonathan.

Une femme hors du temps et de l'espace confrontée plus tard à un mensonge amer, incarné par Jonathan, elle perd l'estime de soi et souffre du regard des autres. Kay, à son tour, se voit comme «la grue » qui vit «sur une patte...», «Comme une girouette » (U.H.D., p.71). Ces images connotent la 
fragilité de Kay devant l'agitation de ses sentiments et son amour déçu pour David, le désiquilibre et la peur de l'inconnu, parce qu'elle l'a aimé follement. Les gens de Fécamp ont dit d'elle qu' «elle est ivre morte», qu' «elle est malade» (p.140), «une sauvage» (p.141). C'est encore une fois une femme brisée comme sa mère: «Je ne savais plus marcher droite et fière comme une allumette»(U.H.D., p.138).

Ce passé ressemble à «une étoile filante». Elle perd aussi sa tranquillité et son sentiment d'être protégée. Elle trouve son refuge dans les livres, après le départ de son frère et de son amant.

Kay s'étonne tout le temps de ce départ: «Sans un mot. Sans une explication. Sans même se retourner »(U.H.D., p. 41). Mais, vers la fin du roman, on découvre le mot laissé par David: «Je pars. Je vous aime tous les deux. Mais j'ai besoin de liberté...Besoin d'être seul. Je ne peux plus être un parmi les trois, je veux être un, tout seul »(U.H.D., p.139). Il essaie de justifier son départ et lui rappelle qu'il produit des films que Kay a déjà vus en France et en a été influencée. Cette production le place sur le chemin de la célébrité dont l'attrait est irrésistible, mais il agit avec égoïsme: «Je n'avais accepté ce boulot que parce que j'étais "en panne" et qu'on me proposait beaucoup d'argent. Que mon nom ferait vendre! » (U.H.D., p.81)

David dit Jonathan est le fils d'un consul américain et a grandi en France. Il connaît le français, l'italien et l'espagnol. Il écrit des livres, des scénarios et produit des films. En réflichissant un peu sur la signification de deux prénoms: David et Jonathan, nous trouvons que la romancière les a bien choisis en dessinant ce personnage. A la lumière de l'analyse faite des prénoms par J.-M. de Foville, le prénom David est chéri des dieux et des hommes (étymologie: de l'hébreu"daoud", aimé, sous entendu de Dieu). Il connote la sensualité, l'intelligence, le trouble, la volonté et la joie de vivre, mais « il n'est pas toujours facile de vivre avec lui ${ }^{\mathrm{xxvii}}$. Depuis trois mille ans, Jonathan, le jeune prince courageux, malheureux était un ami fidèle et très chéri de David $^{\mathrm{xxviii}}$. Dans son analyse, J.-M.de Foville associe les prénoms aux signes du zodicaque, aux pierres précieuses et aux métaux qui relèvent un peu de la magie et confrontent au mystère. Pour Jonathan le métal qui correspond à son prénom est le mercure. Ce seul métal, qui à l'état liquide se vaporise aisément, incarne bien Jonathan le masqué. Alors que l'or correspond au prénom David, à sa richesse et à son acceuil chaleureux de Kay et de son frère; c'est la raison pour laquelle il est désigné par la romancière par «le Veau d'or »(U.H.D., p.133). La douceur de David revient au signe zodical du Bélier et le signe des Gémeaux pour Jonathan symbolise probablement son souhait de revivre avec Kay. Le diamant est la pierre précieuse pour David et Jonathan. C'est ainsi que la romancière montre que les deux prénoms figurent l'entité d'une même personne. Quant au prénom Kay 
donné à l'épistolière, d'ailleurs comme chez les anglais, il convient à cette jeune fille simple.

Reste à dire que si la romancière a tenu à choisir à ses héros de fiction des prénoms qui conviennent à leur nature et à leur tempérament, son plus grand intérêt porte sur leurs lectures. "Dis-moi ce que tu lis, je te dirai qui tu es et comme tu aimes..."semble dire ce roman de K. Pancol. A première vue, les livres apparaissent comme le pivot de l'entretien des deux épistoliers: Kay/Jonathan avides de lectures, dont la correspondance est produite dans le but de s'informer sur des livres et qui deviennent l'elixir de leur relation. Jonathan s'intéresse aux éditions anciennes, il les recherche au Marché aux Puces de New York. Les lectures de Kay incarnant ses goûts personnels, entament un vrai dialogue culturel et amical au début pour se transformer plus tard en un dialogue amoureux. «J'aime voler très haut en lisant ces livres, toute seule dans ma chambre, face à la mer furieuse, les soirs où la tempête siffle comme une sorcière édentée »(U.H.D., p.23). Cette rêverie lui sert de refuge, une ambiance qui la transmet dans un autre monde dans lequel, elle lit et rêve. La lecture intensifie l'imgination de Kay et double sa vie: "J'imagine Robert Browning entrant dans ma chambre et m'enlevant dans ses bras puissants en me récitant des vers! C'est peutêtre pour cela que j'habite dans un port: j'attends le pirate au grand cour qui m'emportera sur son galion! »(U.H.D., p.23) Sans le savoir, Kay rédige cette lettre au pirate de son cœur et non au pirate au grand cœur. Le mot pirate employé déjà par Josepha au début du roman pour présenter l'histoire, peut avoir pour connotation: l'aventure, le pillage et l'illigimité.

\section{L'Amour et l'intrigue épistolière}

Le sujet essentiel de la correspondance dans Un homme à distance ressemble à celui de $\mathrm{La}$ Nouvelle Héloise car il met l'accent sur: « la fonction "émotive" du langage, celle qui est centrée sur le locuteur» ${ }^{x x i x}$. C'est une «exposition de soi » dans un « lieu fantasmé » créée par une langue qui « devient la forme même de son identité: écrire et vivre deviennent synonymes» (G. Palayret et F. Roussel, pp. 133,135,136). La fonction dite "conative" selon F. Calas (p.16) trouve son chemin directement dans les lettres échangées entre Kay et Jonathan; cette fonction désigne une utilisation du langage pour faire pression sur le destinataire, le persuader (ou le dissuader) d'agir de telle ou telle manière. C'est d'ailleurs l'intention de Jonathan qui en réalité ne souhaite rien au monde que de se faire pardonner par Kay: « Kay, je t'en supplie...Dis-moi de venir. Dis-moi que tu m'as pardonné, que tu me pardonneras, que tu pourras me pardonner un jour, dans une éternité» (U.H.D., p.133)

Cette liaison épistolaire basée d'abord sur leurs lectures laisse la place donc aux confidences amoureuses voir le jour. Cet amour peint dans les premières lettres du 
roman en un tableau animé, qui attire notre attention par sa description rythmée « des chants, des confesssions, des abordages, des orages, des prises d'otages », parfumée par «l'encens », idéalisée par son image sublime est « un amour haut comme une cathédrale», mais violent «comme une bordée de pirates » et enfin déprimé et tenaillé entre la "ferveur" et la « souffrance » (U.H.D., p.8). Amour noué à la souffrance décrit comme "magnifique" d'après Josepha, revient à une ancienne histoire dont Jonathan fait allusion en demandant: « Sait-on à vingt ans ou à vingtcinq ans pourquoi on agit ou, pire, on réagit? Pourquoi on confond tout, amour et amourpropre? » (U.H.D., p. 56).

Jonathan sait émouvoir le cœur de la jeune fille. Selon ses propres termes, il s'assimile à «un médecin qui écrit une ordonnance! » (p.26) où il note en post-scriptum, la définition de l'amour: «L'amour n'est pas qu'un ravissement, mademoiselle. L'amour peut être une torture, un traître, un tricheur. Il emprunte tous les discours, tous les costumes, tous les subterfuges» (U.H.D., p.27). Il lui conseille alors de lire Les Liaisons dangereuses, La cousine Bette pour se souvenir que l'amour « avance masqué» (U.H.D., p. 27)

L'Amour pour Kay doit être au-dessus de tout: "fulgurant", "impossible", "impitoyable" et "intransigeant". «Je ne pardonne pas à l'amour qui compromise, qui s'arrange, qui descend sur terre et obéit aux lois idiotes de notre société...» (U.H.D., p.23) Elle définit l'amour à Jonathan en se souvenant de cet homme fugitif et lâche qui a laissé ses empreintes profondes sur sa vie: «L'amour est un grand menteur, un grand dissimulateur. Il vous force à tout donner puis s'en va, repu, ennuyé, à la recherche d'autres cœurs à dévaliser» (U.H.D., p.77)

Son histoire avec David a échoué parce qu'elle n'avait que quinze ans «L'amour est une sainte enfance », «la douceur », « la pureté » (U.H.D., p. 102). Mais à trente deux-ans et quand elle a mûri, elle connaît à présent ce qui est une « science amoureuse », et une « politique de l'amour»(U.H.D., pp.102-3). Elle considère que cette politique est obligée. Pour mieux comprendre, elle interroge Jonathan: « Pourquoi n'aime-t-on pas les hommes qui restent et vous dispensent de subir les atroces souffrances de l'abandon brutal? Pourquoi a-ton besoin de sublimes douleurs pour sceller les grandes histoires d'amour? » (U.H.D.,p.104) Jonathan lui, indique qu'il n'a pas de réponse. Il se définit comme un homme «lâche et fort», «Cruel et doux», «Généreux et calculateur», «Courageux et couard», «J'ai été fier de moi et j'ai eu honte, très honte» avoue-t-il (U.H.D., p.106). Ces caractéristiques contradictoires postulent son portrait complexe.

Étranger et plus âgé qu'elle, Jonathan a été pour Kay une expérience. Par là « la lettre sert de cadre à l'introspection spontanée à laquelle se livre l'épistolière qui découvre les racines profondes de son amour»(F. Calas, p.75). Au début de leur relation, il la voyait comme une 
enfant, et elle se voyait comme une femme qui vit une histoire d'amour très précoce par rapport à son âge. « Tu étais si jeune mais si savante», « Tu m'as rejeté dans le cours de la vie, tu m' as redonné le goût » (U.H.D., p. 149). Décalage de temps, décalage de l'espace: deux lignes parallèles qui ne se rencontrent jamais. Cette aventure désespérée l'incite à préciser les caractéristiques de l'homme de son avenir qui doit avoir les: « mains solides », les « jambes piliers, arrimées dans le sol ». Souffrant de l'ambiguité et de l'escapade de David, Kay souhaite respirer l'air de la pureté avec un cœur transparent avec, un homme « aux mots simples et clairs, au rire franc et sonore », « à l'ambition modeste...» (U.H.D., p.41) Elle avoue qu'elle a peur d'aimer à nouveau un homme qui peut la quitter même si c'est quelqu'un comme Jean-Bernard qui a beaucoup de qualités: «C'est un homme droit, solide et qui me tire de toutes les situations vacillantes, de tous les bilans dangereux» (U.H.D., p.50).

Le nœud de l'intrigue arrive à sa plénitude quand les deux épistoliers parlent de leurs secrets. Ce moment contribue à l'élaboration et au développement de l'intrigue qui nous frappe par sa simplicité. Kay écrit « Je hais la douceur, la tendresse, la passion quand elles ne viennent pas de lui»(U.H.D., p.74). Le cœur apaisé par cette confession qui décrit les premiers émois d'une femme frustrée par le départ inattendu du voisin américain qui les a sauvés, accueillis et délaissés.
K. Pancol met sur scène la différence de nature entre homme et femme et la capacité de chacun à s'épancher. Voici son personnage qui s'épanche et nous confie un secret dans une lettre de neuf pages en donnant des éclaircissements à son épistolier sur "l'âme des femmes": «Les femmes osent confesser les aveux que les hommes enferment à double clé dans leur cœur » (U.H.D., p.38). En faisant la distinction entre la lettre pour la femme et pour l'homme, L. Versini considère que les hommes ont certains jugements en rédigeant leurs lettres: «Entre les mains des héros masculins, la lettre est instrument de séduction; ils savent que "les femmes" en écrivant, se laissent aller, donnent des armes contre elles»(L. Versini, p.146). La conception de Kay de "l'homme" en tant qu'être masculin se heurte à celle "des femmes" pour Jonathan: «Les femmes seules sont des voraces, des cannibales qui m'effraient» (U.H.D., p.64). Dans la même lettre, il décrit Kay comme une « femme affamée ». Elle confirme en se décrivant comme une femme farouche, mais qu'elle se voit en même temps comme une femme "déterminée" et elle avoue à Jonathan que « Rien ne me fera plier. Ni votre projet, ni vos ordres, ni vos menaces » (U.H.D., p.82).

Quand Kay se révolte, elle se donne une arme contre son correspondant. Sous l'emprise des écrivains et de la lecture, elle sent sa faiblesse. Son épanchement se fonde sur la définition de la lecture: « La lecture n'est pas une activité innocente. On n'en ressort pas toujours 
indemne. La lecture est dangereuse. Elle m'a extirpé des aveux que je ne vous aurais pas faits sous l'emprise du bourreau» (U.H.D., p.105). Ceci nous amène à déduire que la trame romanesque du roman épistolaire est complexe: toutes les fonctions de la lettre à titre d'exemple informer, toucher, tromper se fusionnent ensemble pour exposer une situation d'abord ambiguë mais qui se démystifie petit à petit (S. Gruffat, p.104).

\section{E. Le péché épistolaire et le dénouement de l'intrigue}

La fiction revêt au personnage un masque. Comme l'indique F. Calas: «Le roman par lettres est l'art du masque, qui colle à la peau de la vie elle-même» (F. Calas, p.23). Jonathan revisite le passé avec un autre œil; il reconnaît que son «ambition avait tout brûlé » (U.H.D., p.150). Il réussit à affronter son moi, mais il cherche des pistes et des moyens qui lui facilitent l'accès au cour de Kay. Mais en essayant de se rapprocher d'elle, il commet une autre faute en se camouflant: «Alors j'ai pris un masque et ce masque m'a défiguré. Ce masque était pitoyable et lâche. Pire! Ce masque était peut-être ma nouvelle identité...» (U.H.D., p.150) Jonathan choisit la lettre comme moyen de retour à Kay. L'intrigue se noue alors autour de ce camouflage. Le double déguisement d'identité et de métier de David/Jonathan renforce le côté fictif du roman. La distance est abolie et l'action donne plus d'actualisation au sentiment. Pour déclarer cet aveu, la romancière a recours à plusieurs atouts: d'abord l'allusion au secret par des romans épistolaires, le suspense du roman policier, les films et l'exploitation du lieu.

Dans la troisième lettre, Kay parle de Maison des autres d'un auteur italien Silvio d'Arzo et met l'accent sur une lettre jamais envoyée. Cette œuvre littéraire donne l'occasion à retarder l'aveu des secrets, elle arrive par ce titre à évoquer la curiosité du lecteur: «Il ne faut jamais dire les secrets si l'auteur n'est pas préparé, s'il n'est pas passé par les mêmes tourments que vous» (U.H.D., p.16) écrit Kay. Jonathan avoue: «Je savais que ce livre me secourrait... Mais je ne m'attendais pas à une telle violence» (U.H.D., p.55) Les mots de Kay réveillent sa conscience et il éprouve des remords. Elle réussit à desceller son cœur et à ouvrir la boîte noire du passé. Ne pouvant pas avouer directement sa vérité, Jonathan a recours à d'autres écrivains.

Jonathan avide de beaux livres, surtout de romans et de films comme celui de François Truffaut: Les Deux Anglaises et le continent; il va jusqu'à préférer les héros de fiction aux êtres réels, à tel point qu'il avoue qu'il a toujours eu le désir de se glisser entre les pages des romans afin de forcer les personnages à se parler. Il devient lui aussi un personnage fictif. Il présente sa mission: rédiger le guide des endroits de charme en France pour un éditeur américain. Néanmoins, il avoue à Kay, dans une autre lettre, qu'il déteste les guides comme elle: «Je préfère le 
quotidien, le détail volé, un mot de patois, une frite dorée, un coucher de soleil simple comme un trait d'orange, le piqué d'une mouette sur un filet, un bonimenteur dans un supermarché» (U.H.D., p.52). La contradiction de ses paroles perturbe Kay.

Elle se pose deux questions fondamentales qui sont à la base d'Un homme à distance: «Est-ce que les livres sont un moyen de tout se dire, même l'inavoué, le plus terrible secret?» (U.H.D., p.38) Ces deux questions nous renvoient à l'identité d'un homme qui vit loin d'une femme qu'il connait depuis plusieurs années et dont celle-ci n'a jamais pu découvrir sa vraie identité au début de leur correspondance. Vers la fin du roman, elle devine même qu'il s'agit d'un journaliste, incité par sa curiosité à dévoiler les secrets de la vie de David. Elle ne comprend sa véritable nature, ni à quinze ans, ni après leur correspondance.

Dans la quatrième lettre, il parle d'un auteur italien: Erri de Luca. Il apprécie la littérature italienne qui renferme des trésors, mais n'est pas assez considérée dans le monde des belleslettres. De cette littérature, il aime: Le fils de Bakounine de Sergio Atzeni. Jonathan estime la littérature française et Font-Romeu en est un de ses ouvrages préférés: «petit bijou de la littérature française »(U.H.D., p.61). Ce livre composé de quatre-vingt-huit pages est décrit comme «un de ces livres qui vous marquent au fer rouge et dont on ne se relève jamais! », Qui vous collent à la peau et vous accompagnent partout comme un fantôme affectueux! »(U.H.D., p.62)

Ensuite, après cinq mois de "l'énigme Jonathan", l'hésitation et la peur de la récation de Kay incitent l'américain à se poser des questions: "Peut-on être pardonné avant qu'il ne soit trop tard? Ai-je dépassé le temps autorisé? Vais-je mourir avant que de parler? »(U.H.D., p. 55) Dans sa treizième lettre, l'aveu est déclaré graduellement: «L'aveu me monte aux lèvres, Kay, et si je le retiens, c'est que j'ai peur encore. »(U.H.D., p.67). Il fait allusion au passé qui a duré plus de quatre ans: « Cet amour impossible. Et la vie qui recouvre tout de son ciment d'indifférence, d'appétence, d'ambition puérile, factice, facile » (U.H.D., p. $68)$.

L'existence d'une fiction mitigée du réel enrichit le roman épistolaire. Les correspondants sont des personnages inventés comme ceux des Lettres Persanes, mais on les reconnaîtrait facilement dans notre réel qui est souvent envahi par des amiguités, des mystères ou des Faux-monnayeurs comme le dit Kay. Un autre personnage "fauxmonnayeur", à part Jonathan, est Nathalie qui "entre en scène. Comme les confidentes de Racine ou les servantes de Marivaux". Elle a caché à Kay la vraie identité de Jonathan «si beau, si charmant, si séduisant» (U.H.D., p.125). Elle craignait que Kay ne retombe encore une fois dans « un amour impossible». Elle comptait la protéger car elle savait que 
cette dernière n'aimait pas «raisonnablement» (U.H.D., p.62). Nathalie en travistissant Jonathan a en quelque sorte sauvé Kay par sa sagesse mystérieuse. Cependant, son rôle, comme dans le roman policier, ressemble à celui du coupable dont on ne se méfie point et qu'on demystifie son secret à la fin de l'histoire. En effet, les livres policiers qu'elle «dévore» (p.22) lui font agir de la sorte et Kay voyait «qu'il fallait qu'elle arrête de lire des romans à quatre sous, ça lui pourrissait la tête! »(U.H.D., p.43) La romancière par cette stratégie de suspense donne un autre plaisir à la lecture au roman épistolaire.

Dans sa vingt-sizième lettre, composée de six pages, Jonathan alias David raconte à Kay l'histoire de sa vie. Cette lettre provoque la colère de Kay et l'incite à lui poser une série de questions dont: " Qui êtes-vous?" répétée trois fois, puis elle le cible de propos indignes: «Qui êtes-vous pour enfoncer les portes de ma vie, violer mon intimité, saccager mes souvenirs? », «Un écrivain en mal de matière fraîche, de meat, comme vous dites, qui se nourrit de la vie des autres parce qu'il est stérile comme une figure sèche?» (U.H.D., p.122) Elle soupçonne David d'avoir confié des informations à une personne lui demandant de prendre de ses nouvelles. Poussée par la rage et la colère, elle lui demande de lui adresser ce mot: «fuck off» ou pour parler comme le vieux forçat: «Les hommes. Font chier» (U.H.D., p.126): Kay a refusé une importante somme d'argent pour confier des informations sur David à un journaliste de Vanity Fair. Les gros titres des journaux parlent de lui comme d'un être mystérieux, inconnu mais distingué et connu au cinéma américain. Devant toutes ces hypothèses, l'épistolière française troublée, oscille entre le bonheur du temps passé, la douleur du départ imprévu et l'ambiguïté de l'épistolier américain qui impose à sa vie une domination éclatante. Il avoue qu'il l'aime "à la folie" et que « L'amour était devenu trop d'amour » (U.H.D., p. 147). Face au sentiment de culpabilité, il a recours aux vers d'Emily Dickinson:

«Le "pourquoi" navré de l'amour

Est tout ce que l'amour peut dire

De deux syllabes sont bâtis

Les plus vastes cours qui se brisent» (U.H.D., p.72).

Kay répond en citant le même auteur mais en utilisant des traits d'union et non des points: «Si pouvoir----équivalent à vouloir---

Ténu serait----le Critère---

C'est l'ultime de la Parole---

Que l'Impuissance à Dire » (U.H.D., p.75).

Après un an de correspondance, il incite Kay à se rappeler de sa première lettre: «Je l'ai relue, ces derniers jours. Oh! Je voudrais tant revenir à ces jours heureux où vous m'ouvriez. votre librairie, votre tête, votre cœur» (U.H.D., p.132). Nous remarquons que le ton de regret et d'amertume domine la première lettre signée "David" ayant pour fin: « Tout est de ma faute», «Que pourrais-je faire pour me racheter? », «Le Veau d'or fracassé» (U.H.D., 
p.133). Après avoir connu le succès et après la mort de son père, il sent la solitude et avoue son regret à Kay: «j'ai tout, mais je n'ai rien puisque je t'ai perdue »(U.H.D., p.147). Avec le dénouement de l'intrigue, quand le masque de Jonathan tombe, le lecteur découvre l'aveu du péché épistolaire. Après cet aveu, Jonathan réapparaît sous sa véritable identité. Mais il ne réussit point à réconquérir son épistolière. $\mathrm{Ce}$ moment bouleverse Kay: «Vous m'aviez apprivoisée avec vos lettres, avec vos mots, vos formules, vos images, vos narrations. Comme le renard apprend au Petit Prince à l'apprivoiser» (U.H.D., p.124) Cette deuxième chute à trente deux ans est plus dure que la première. Mais elle a plus de distance cette fois, elle comprend mieux Jonathan. Elle enumère ses défauts «Votre brutalité », « votre mensonge », «votre habileté de fauxmonnayeur ont tout détruit»(U.H.D., p.124). Par ailleurs, l'ombre de suicide de son frère Marco reste un obstacle entre eux. Après un grand silence, Kay envoie sa dernière et sa plus longue lettre de treize pages. Elle insiste sur le fait qu'elle écrit pour la dernière fois: « Lis-la, relis-la, encadre-la, place-la sur ta cheminée à côté de tes oscars d'homme triomphant». Elle termine sa lettre en citant Madame de Staël «"La gloire est le deuil éclatant $d u$ bonheur"» (U.H.D., p.135). Le silence est plus éloquent que la parole. C'est pour cette raison que les deux épistoliers arrêtent de s'écrire et que Kay se débarasse des lettres. Le lecteur peut deviner la séparation définitive et l'incapacité de Kay de le tolérer. Une autre stratégie de suspense à lauqelle a recours la romancière est l'exploitation du lieu. De l'hôtel Le Pigeonnier, Jonathan tire encore le fil conducteur de l'intrigue. Ne pouvant pas avouer sa vraie identité, David lui envoie la cassette du film Les Ensorcelés en version française; The Bad and the Beautiful en anglais de Vincente Minnelli: «Ce sera ma manière à moi de me confesser à vous », «Je suis the bad and the beautiful...» (U.H.D., p.127). Le recours à ces films célèbres permet au lecteur de visualiser cet aveu. D'autre part, K. Pancol introduit une part réelle dans le roman en mentionnant le nom du cinéariste américain Vincente Minnelli.

Les épistoliers ne s'écrivent plus. Jonathan continue sa production cinématographique à succès et Kay passe son temps à essayer de résister aux vagues de la mer, ce dont Josepha est témoin: "j'assiste, désolée, à la furie des vagues qui la font sauter comme un bouchon, qui la plaquent, qui la roulent, qui l'écorchent, la font disparaître puis la rejettent sur les galets »(U.H.D., p.152). En voyant ses photos dans les journaux, Kay avoue qu'elle ne reconnaît plus cet homme «mort», « qui avait trahi », «qui se mentait à lui-même » (U.H.D., p.123). Elle avoue: «Je ne l'aime plus. J'ai aimé, autrefois, un homme qui s'appelait David et que je respectais et que j'admirais follement»(U.H.D., p.123). C'est ainsi que le 
rythme du roman balance entre la scène qui « donne l'illusion d'une cöincidence parafite entre le temps qu'on met à lire l'épisode et le temps qu'il met à se dérouler» (V. Jouve, pp.36, 37) (avant l'aveu), la pause (temps du silence) et le sommaire (lettre de l'aveu et réponse de Kay).

Une question s'impose alors dans le roman épistolaire de K. Pancol ces stratégies de suspense se terminent-elles par le rapprochement ou l'éloignement des personnages?

Kay souffre des douleurs de la séparation tout au long de sa vie: le souvenir d'une mère maltraitée par le père, puis sa mort, le remariage de son père, le départ de la maison paternelle, le départ de Jonathan, le suicide de son frère et enfin, la découverte du déguisement de David sous le nom d'emprunt de Jonathan. Après quatre ans d'amour, une qinzaine d'année d'absence et un an de déguisement, Jonathan a imaginé que Kay va céder facilement. Elle parle plus à notre cœur, nous pouvons même nous apitoyer sur son sort tragique et l'on déduit le vrai message communiqué par l'auteur: «La seule vérité dont on puisse traiter est celle du cour, suggère Rousseau, ce qui revient à dire que la vérité appartient au seul sujet créateur et sa crédibilité à sa puissance d'illusion sur le lecteur $»^{\mathrm{xxx}}$.

\section{Le lecteur de droit/impliqué et le lecteur externe/virtuel}

Dans le roman épistolaire, nous trouvons, deux types de réception: une réception intérieure entre les deux épistoliers dont l'un et l'autre joue le rôle du lecteur-récepteur des lettres et une réception extérieure par le lecteur du roman.

Sous l'angle de l'acte d'écriture et celui de lecture, la relation triangulaire entre destinataire ou «émetteur », destinateur ou premier « récepteur », ou « récepteur précis» et lecteur « récepteur second (le lecteur)» ou « voyeur ou de tiers indiscret » est désignée par S. Gruffat comme «enchâssement des situations de communication »(pp.4, 12,13). Quant à F. Calas, il distingue entre deux lecteurs: le lecteur de droit ou impliqué ou encore le destinataire direct (le personnage) et le scripteur ou le lecteur externe ou virtuel ou encore le destinataire indirect. Le lecteur de droit est le « destinataire des lettres à l'intérieur de l'espace de la correspondance privée » (F. Calas, p.56).

L'existence du premier ou principal lecteur semble un piège voulu par la fiction. Pourquoi? « L'auteur de la forme épistolaire s'amuse à déjouer nos capacités de lecture et à amoindrir notre rôle pour nous imposer une participation impure »(F. Calas, p.66). D'où se tisse «la dimension pragmatique », selon le terme de F. Calas, du rapport existant entre le texte et son lecteur. Selon le même auteur, c'est une des spécificités du roman épistolaire. 
Ce témoin des réactions des épistoliers ou ce lecteur virtuel peut entrer de plein-pied dans l'intimité des correspondants parce que les épistoliers vivent leurs vies au temps qu'ils écrivent leurs lettres. Il est imprégné par l'effet de la communication entre les deux épistoliers. L'écriture épistolaire évoque la curiosité du lecteur externe durant les moments de silence entre les deux épistoliers afin de combler les lacunes de leurs absences. C'est ainsi que ce lecteur devient ami clandestin des épistoliers, voire par la vraisemblance de la fiction un pacte de lecture entre l'auteur et son lecteur présente la correspondance comme une expérience vécue (Cf. S. Gruffat, p.31).

La fiction épistolaire dépend de l'interprétation du lecteur: «il bénéficie d'une omniscience qui lui permet d'apprécier les réactions des personnages, de repérer le schéma actantiel*, d'anticiper le déroulement de l'intrigue» (S. Gruffat, p.100). Aussi le lecteur peut-il toucher de près les fluctuations, les variations et les développements des sentiments de chacun (Cf.S. Gruffat, p.90). Différent des autres types de roman, le roman épistolaire est plus proche du lecteur. Cette technique permet la transmission des idées de l'auteur d'une manière tout à fait directe. Ceci est dû à la « proximité de la voix des personnages », et à leur «grande autonomie» mises sur scène par «le retrait de l'auteur » (F. Calas, p.112). Le message est transmis au lecteur virtuel par le lecteur impliqué.
Donc, en faisant ainsi le lien entre le lecteur et le «type de lecture », nous pouvons déduire que:

- Le lecteur impliqué entame une «lecture incluse» (S. Gruffat, p. 5), « Le lecteur possède un savoir qui se limite à ce que le destinataire peut retirer d'une lettre qu'il reçoit» (F. Calas, p.57).

- Le lecteur virtuel entame une lecture «dans un espace extérieur» (G. HarocheBouzinac, p.5). Le lecteur français ou étranger a, sous ses yeux, les épistoliers et le romancier. Il connaît un mouvement de va et vient entre les deux épistoliers qui entament une lecture mutelle.

N'oublions pas aussi de distinguer un troisième type de lecture dite "médiative" ou "moraliste" basée sur le dégagement des témoignages « d'un savoir anthropologique sur les conduites humaines, en modélisant certaines attitudes de vie »(G. Haroche-Bouzinac, pp.5-6). A titre d'exemple: les écrits de voyage de Jonathan projettent la valeur d'un journal de voyage. Selon A. Viala, la relation épistolaire a trois interlocuteurs: deux interlocuteurs individualisés et un interlocuteur multiple et anonyme: le public, autrement dit, les lecteurs réels ou potentiels de l'ouvrage ${ }^{\mathrm{xxxi}}$. Cette relation se fonde sur deux plans d'énonciation: les partenaires de la correspondance et le public témoin de ceux-ci et de leurs textes. Cette situaton est homologue à celle du théâtre, où une énonciation s'accomplit sur la scène et devant le public. 
Autour de la classification des genres et des publics des lecteurs, certains critiques comme L. Versini considère que l'écriture épistolaire et son public de lecteur est: «Consacré aux femmes, composé très souvent par des femmes, le roman par lettres a pour premier public les femmes » (L. Versini, p.60) Quoi qu'il soit homme ou femme qui lit ou écrit, nous ne préférons pas la catégorisation des genres, parce que tout simplement cette classification nous paraît arbitraire.

En fin de compte une question peut surgir sur la postérité du roman épistolaire, connaît-elle son épanouissement ou son déclin? A notre époque, où la presse est dominée par le progrès des multimédias (téléphone portable et internet), la communication devient rapide et facile. Les gens tendent de plus en plus à se parler et l'écriture tend à réculer. Les personnages du roman utilisent des formes modernes de commuication: l'écriture sur ordinateur, et le courrier éléctronique. Malgré ce progrès, S. Gruffat insiste sur l'importance de la lettre: «Il semblerait même qu'en cette époque de presse, le fait d'écrire une lettre constitue un acte plus significatif et moins banal que jamais » (S. Gruffat, p.116).

\section{Conclusion}

A notre avis, le roman épistolaire n'est pas une forme "hybride" ou "ambiguë", ou "floue". C'est "un genre protéiforme" qui englobe, le roman sentimental par l'épanchement profond, le roman policier par le suspens, le roman- confidence, le roman-journal, en un mot, roman épistolaire-journal qui a une vocation sentimentale et littéraire.

Par leurs écrtitures, les personnages-épistoliers se constituent, par leurs voix ils se mettent sur sècne devant le lecteur extérieur par leurs portraits et leurs réflexions. La réflexion et la méditation des épistoliers s'avère lorsqu'ils se posent des questions tel: "Que laisseronsnous de nous en guise d'épitaphe? » (U.H.D, p.21), question posée par Jonathan. La reprise en leitmotiv de lectures, d'observations critiques sur les livres lus est la pierre angulaire du dialogue entre les deux épistoliers. Pour Kay, l'épistolière bouleversée, "allumette", "girouette", la lecture est "dangereuse", parce que «les livres sont un moyen de tout se dire: l'inavoué, le plus terrible secret? »(U.H.D, p.38) A la fin du roman le duo épistolaire se sépare, mais il nous reste les traces de leurs échanges: les lettres.

Signalons que l'originalité de l'écriture épistolaire à laquelle a procédé K. Pancol s'accentue par la cohésion et la continuité dûe à l'effet de la circularité des particularités formelles, thématiques et techniques. Circulartité par l'ordre chronologique, par le classement de chaque lettre en face de sa réponse, circularité entre l'exorde et la conclusion, circularité par le retour des propos échangés entre épistolier et épistolière de nationalités différentes, circularité des voix fait le grand succès de cette narration à deux voix. 
Même cette voix-off concrétisée par la voix de Josepha joue en cercle. Elle débute la narration «L'important, ce sont les lettres » et la boucle: «Elle (Kay) doit lire. Ou écrire une lettre qu'elle ne postera jamais ». Circularité entre le passé des souvenirs, le présent de l'écriture, le futur des retrouvailles et la réaction des futurs lecteurs. Circularité entre la lecture et l'écriture: les épistoliers lisent et citent dans leurs lettres des auteurs de différentes nationalités, circularité par un dialogue entre les civilisations, tissée à l'intérieur d'un discours de soi à soi, et par une comparaison entre les Français et les Américains, met en scène un traitement technique original $\mathrm{du}$ roman épistolaire et notamment celui de l'intrigue nouée autour de deux chocs, de deux chutes. Le départ imprévu de Jonathan, le déguisement, l'emploi de la lettre comme masque, l'exploitation de l'espace pour en tirer le fil des souvenirs et enfin la déclaration de l'aveu par des films en anglais et en français envoyés sur cassette font $\mathrm{du}$ tissage romanesque un tout cohérent et vraisemblable.

\section{Bibliographie selective}

\section{Corpus}

PANCOL Katherine, Un homme à distance, Albin Michel, Paris, 2002*.

\section{Ouvrages sur le roman épistolaire}

- CALAS Frédéric, Le Roman épistolaire, Collection "128", Nathan Université, Paris, 1996.
- GRUFFAT SABINE, L'épistolaire, Ellipses Marketin, Paris, 2001.

- HAROCHE-BOUZINAC Geneviève,

L'épistolaire, Hachette, Paris, 2002.

- HUBIER Sébastien, Littératures intimes, les expressions dumoi, de l'autobiographie à l'autofiction, Armand Colin, Paris, 2003.

- JACKSON John, RIGOLI Juan et SANGSUE Daniel, Être et se connaître: littérature et sciences Humaines, préface d'Alain Corbin, Métropolis, Genève, 2006.

- OMACINI Lucia, Le roman épistolaire français au tournant des Lumières, Honoré Champion, Paris, 2003.

- PALAYRET Guy et ROUSSEL François, L'échange entre réciprocité et transaction, Belin, Paris, 2002.

- VERSINI Laurent, Le Roman épistolaire, Collection: "Littératures modernes", Presses Universitaires de France, Paris, 1979.

\section{Ouvrages généraux}

- DE FOVILLE Jean-Marco, Les pronoms de vos enfants: origine, histoire, signification, Hachette, Paris, 1995.

- -DUCHENE Roger, Naissance d'un écrivain, Fayard, Paris, 1996.

- GENETTE Gérard, Poétique, Seuil, Paris, 1987.

- JOUVE Vincent, La poétique du roman, Armand Colin, Paris, 2001.

- -LEJEUNE Philippe, L'autobiographie en France, Armand Colin, Paris, 2003.

- MACHEREY Pierre, A quoi pense la littérature? Presses Universitaires de France, Paris, 1990. 
- SAYED Fathi, La syntaxe et l'affectivité Les amis du livre-Éditeur, Le Caire, 2000.

\section{Articles}

- DELON Michel, Les correspondances d'écrivains, in Magazine litéraire, $\mathrm{n}^{\circ} 422$, mai 2005, p. 30.

- GRASSI Marie-Claire, Des lettres qui parlent d'amour, in Romantisme, n8,1990, p. 23

- LACOUE-LABARHE Isabelle, Lettres et journaux de femmes entre écriture contrainte et affirmation de soi, in Tumultes, $\mathrm{n}^{\circ} 36,2011$, pp.113-132.

- MONTENOT Jean, Ce que révèle la correspondance des écrivains, in Lire, $\mathrm{n}^{\circ} 355$, été 2007, pp 30-33.

\section{Dictionnaire et encyclopédie}

- BASSIÈRE Jean, BLOCHE Michelle et al, Littérature et genres littéraires, Encyclopoche Larousse, Larousse, Paris, 1978.

- Encyclopoche Larousse, Larousse, Paris, 1978.

- Le Grand Dictionnaire encyclopédique, éd. Larousse, Paris, T. 4, Larousse, Paris, 1983.

- ROBERT Paul, Petit Robert, éd. Le Robert, Paris, 1991.

\section{Sitographie}

- $\quad$ http://www.katherine-pancol.com/ecrivez-moi

- $\quad$ http://www.universalis-edu.cim/index.php

- $\quad$ http://fr.wikipedia.org/wiki/autofiction

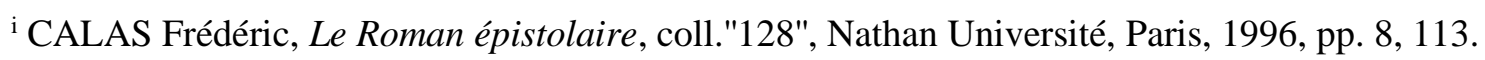

ii «L'adjectif "épistolaire", que le langage critique a substantivé pour désigner l'ensemble des facteurs liés à l'échange de lettres, est un emprunt au latin médiéval epistolarium qui désigne un livre de lettres. Il qualifie tout ce qui "a rapport à la correspondance par lettres"» HAROCHE-BOUZINAC Geneviève, L'épistolaire, Hachette, Paris, 2002, p.3.

iiiVERSINI Laurent, Le Roman épistolaire, Coll."Littératures modernes", PUF, Paris, 1979, p.69. Voir aussi Le Grand Dictionnaire Encyclopédique Larousse, Larousse, Paris, 1983, p.3828.

${ }^{\text {iv }}$ LEJEUNE Philippe, L'autobiographie en France, Armand Colin, Paris, 2003, p. 43.

${ }^{v}$ MACHEREY Pierre, A quoi pense la littérature? Presses Universitaires de France, Paris, 1990, p. 77.

${ }^{v i}$ ROUGEOT Jacques, La littérature épistolaire, Littérature et genres Littéraires", Encyclopoche Larousse, Larousse, Paris, 1978, p.169. Il indique que l'habitude de publier des correspondances se répandit au XVI ${ }^{\mathrm{e}}$ siècle.
}

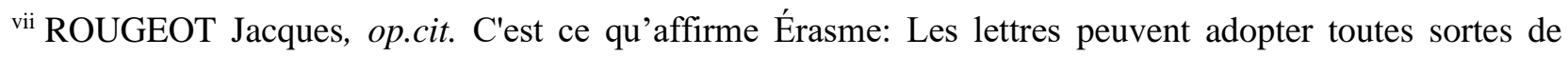
formes et de sujets, mais il maintient une rhétorique de la monovalence: un sujet, un propos et une lettre.

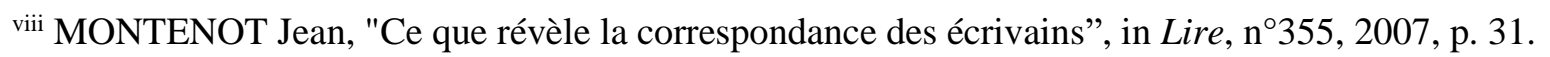

ix PALAYRET Guy et ROUSSEL François, L'échange entre réciprocité et transaction, Belin, Paris, 2002, p.136. 
${ }^{\mathrm{x}}$ GRASSI Marie-Claire, "Des lettres qui parlent d'amour", in Romantisme, nº, 1990, p. 23. Il est à mentionner que le statut de la confidentialité de la lettre a grandement changé avec le temps. Au Moyen âge «Un message réellement confidentiel aurait été de préférence transmis oralement, et l'on suppose même que les lettres se limitaient à des instructions données pour mémoire au messager» (G. HarocheBouzinac, p.14). Plus tard, la représentation des sentiments n'est plus symbolique, mais on écrit librement.

${ }^{x i}$ http://www.katherine-pancol.com/ecrivez-moi. «Roman vendu à plus d'un million d'exemplaires et traduit en russe, chinois, ukrainien, italien, polonais, coréen, vietnamien et néerlandais». A l'âge de 25 ans, K. Pancol publie son premier roman Moi d'abord. En 1981, grâce à un deuxième roman La Barbare, elle devient écrivain à plein temps. Lauréate en 2006 du prix "Maison de la Presse" pour son roman Les yeux jaunes des crocodiles, les éditions Gorodets Publishing lui attribuent l'année suivante, le prix du meilleur auteur. Sa réussite continue grâce à la publication de ses romans aux éditions du Seuil, Albin Michel et Fayard.

${ }^{\mathrm{xii}}$ http://www.katherine-pancol.com/ecrivez-moi

xiii GRUFFAT Sabine, L'épistolaire, Ellipses Marketin, Paris, 2001, p. 89.

${ }^{\text {xiv }}$ http://www.universalis-edu.cim/index.php. La naissance du roman par lettres proprement dit peut être datée de 1669, avec les Lettres de la religieuse portugaise.

${ }^{x v}$ Cf. GENETTE Gérard, Poétique, Seuil, Paris, 1987, pp. 10-11.

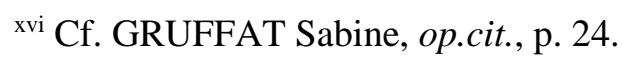

xviiJOUVE Vincent, La poétique du roman, Armand Colin, Paris, 2001, p.14.

xviii JOUVE Vincent, op.cit., p.26.

${ }^{x i x}$ PANCOL Katherine, Un homme à distance, Albin Michel, Paris, 2002, p.7. Nous allons utiliser l'abréviation U.H.D. pour Un homme à distance.

xx PALAYRET Guy et ROUSSEL François, op.cit., p. 133.

xxi L'en-tête du type: "Mon cher, Ma chère", que nous connaissons aujourd'hui, n'est pas systématiquement en usage à l'époque classique. Cette formule est intégrée au corps même de la lettre, de laquelle elle ne se sépare pas par l'espace blanc, de mise aujourd'hui» HAROCHE-BOUZINAC Geneviève, op.cit., p.20.

xxii http://www.universalis-edu.cim/index.php.

xxiii Voir JACKSON John, RIGOLI Juan et SANGSUE Daniel, Être et se connaître: littérature et sciences humaines, Métropolis, Genève, 2006, préface d'Alain Corbin.

${ }^{\text {xxiv }}$ Cf. SAYED Fathi, La syntaxe et l'affectivité, Les amis du livre-Éditeur, Le Caire, 2000, p.30. 
${ }^{\mathrm{xxv}}$ Fécamp est une commune française de Haute-Normandie, ancien port morutier, dans le département de la Seine-Maritime. http://fr.wikipedia. org/wiki.

xxvi OMACINI Lucia, Le roman épistolaire français au tournant des Lumières, Honoré Champion, Paris, 2003, p. 39.

xxvii DE FOVILLE Jean-Marco, Les pronoms de vos enfants : origine, histoire, signification, Hachette, Paris, 1995, p. 223.

xxviii Ibid., p. 386.

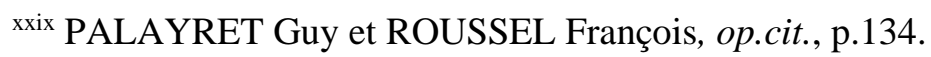

${ }^{\mathrm{xxx}}$ OMACINI Lucia, op.cit., p. 22.

${ }^{*}$ Système des personages qui jouent des rôles divers au sein du récit (S. Gruffat, p. 124).

${ }^{\mathrm{xxxi}}$ http://www.universalis-edu.cim/index.php

* Étant donné que ce titre se répète souvent, nous le désignons par l'bréviation U.H.D. 